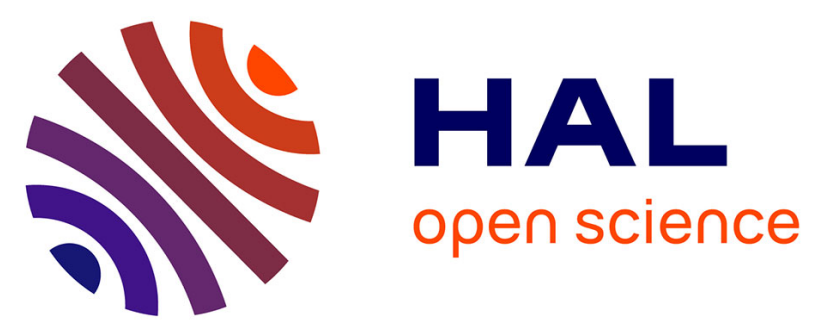

\title{
Influence d'une hétérogénéité macroporale sur les processus de transport de soluté dans un milieu poreux : expérimentations sur sols modèles et simulations par la méthode de Lattice-Boltzmann
}

\author{
Stéphane Batany, Pierre-Emmanuel Peyneau, Béatrice Bechet, Laurent \\ Lassabatère, Pamela Françoise Faure, Patrick Dangla
}

\section{To cite this version:}

Stéphane Batany, Pierre-Emmanuel Peyneau, Béatrice Bechet, Laurent Lassabatère, Pamela Françoise Faure, et al.. Influence d'une hétérogénéité macroporale sur les processus de transport de soluté dans un milieu poreux: expérimentations sur sols modèles et simulations par la méthode de Lattice-Boltzmann. La Houille Blanche - Revue internationale de l'eau, 2017, 3, pp.32-38. 10.1051/lhb/2017019 . hal-01719399

\section{HAL Id: hal-01719399 \\ https://univ-lyon1.hal.science/hal-01719399}

Submitted on 6 Nov 2018

HAL is a multi-disciplinary open access archive for the deposit and dissemination of scientific research documents, whether they are published or not. The documents may come from teaching and research institutions in France or abroad, or from public or private research centers.
L'archive ouverte pluridisciplinaire HAL, est destinée au dépôt et à la diffusion de documents scientifiques de niveau recherche, publiés ou non, émanant des établissements d'enseignement et de recherche français ou étrangers, des laboratoires publics ou privés. 


\title{
Influence d'une hétérogénéité macroporale sur les processus de transport de soluté dans un milieu poreux : expérimentations sur sols modèles et simulations par la méthode de Lattice-Boltzmann
}

\author{
Stéphane BATANY ${ }^{1}$, Pierre-Emmanuel PEYNEAU ${ }^{1}$, Béatrice BECHET ${ }^{1}$, Laurent LASSABATERE², Paméla \\ FAURE $^{3}$, Patrick DANGLA ${ }^{3}$
}

\author{
${ }^{I}$ LUNAM Université, IFSTTAR, GERS, EE, F-44340 Bouguenais, France-e-mail : pierre-emmanuel.peyneau@ifsttar.fr \\ ${ }^{2}$ Université de Lyon, UMR 5023 Laboratoire d'Ecologie des Hydrosystèmes Naturels et Anthropisés, Université Lyon 1, ENTPE, CNRS, 3, rue \\ Maurice Audin, F-69518 Vaulx-en-Velin, France-e-mail : laurent.lassabatere@entpe.fr \\ ${ }^{3}$ Université Paris-Est, Laboratoire Navier, UMR 8205, CNRS, Ecole des Ponts ParisTech, IFSTTAR, F-77455 Marne-la-Vallée, France- \\ e-mail : patrick.dangla@ifsttar.fr
}

\begin{abstract}
RÉSUMÉ. - La modélisation des écoulements et du transport dans les milieux poreux est un domaine actif pour le suivi de la contamination dans les sols ou les bassins de rétention notamment. La prise en compte d'hétérogénéité de type macroporale peut souvent être nécessaire, et pour cela de nombreux modèles numériques utilisent des concepts de double ou multi-perméabilités afin de rendre compte des différentes perméabilités d'un même milieu. Cependant, les modèles classiques semblent sous-estimer le rôle de chemin préférentiel d'un macropore, car le champ d'action de celui-ci doit être élargi dans la matrice poreuse pour ajuster au mieux les données expérimentales. Cette étude se propose de comprendre les mécanismes d'échange hydraulique et de masse entre le macropore et la matrice poreuse, en utilisant un milieu poreux modèle de billes de verre, et en utilisant la méthode de Boltzmann sur réseau pour simuler des écoulements. Les premiers résultats montrent que le débit d'injection influe sur l'apparition d'une inflexion des courbes de percée d'un traceur. Les résultats indiquent que la diffusion, plus forte dans ce cas, provoque des transferts de masse importants vers la matrice poreuse. De plus, les simulations montrent que l'écoulement préférentiel est étendu dans la matrice poreuse sur une zone de même dimension que le diamètre moyen des grains. Ces résultats montrent que l'influence du macropore doit être étendue dans la matrice poreuse pour l'écoulement et que la zone d'échanges diffusifs latéraux dépend du coefficient de diffusion du traceur.
\end{abstract}

Mots-clés : Écoulement, courbe de percée, diffusion moléculaire, macropore artificiel, méthode de Boltzmann sur réseau, transport préférentiel, simulation numérique, résonance magnétique.

\section{Macroporal heterogeneity influence on solute transport process in porous media: experiments on model soil and modeling with Lattice-Boltzmann method}

\begin{abstract}
Flow and solute transport modeling in porous media is an important issue for the monitoring of pollutant contaminations in soils or retention ponds. It is often necessary to take into account macroporal type heterogeneities. For this purpose, several numerical models use double or multi permeability concepts in order to characterize different permeabilities of a porous medium. However classical models seem to underestimate the impact of the preferential path induced by a macropore. This impact has to be extended in the surrounding porous matrix for the good fitting of experimental data. This study proposes to understand hydraulic and mass exchange mechanisms between the macropore and the porous matrix, by using a model porous media made of glass beads, and by using the lattice-Boltzmann method for flow simulations. First results show the influence of flow rate on the apparition of an inflection on breakthrough curves. Results show that molecular diffusion, higher in this case, causes higher mass transfer from the macropore to the porous matrix. Moreover, simulations show that the preferential flow is extended to the porous matrix in a zone having the same dimension than the macropore. These results emphasize that the macropore influence has to be extented in the porous matrix for flow and the diffusive exchange zone depends on the molecular diffusion coefficient of the tracer.
\end{abstract}

Key-words: flow, breakthrough curves, molecular diffusion, artificial macropore, lattice-Boltzmann, preferential flow, numerical modeling, magnetic resonance.

\section{INTRODUCTION}

Le transport de polluants dans les milieux poreux est un enjeu majeur dans nos sociétés, avec pour principal aspect concret la question de la qualité des eaux souterraines. Dans les milieux urbanisés, des substances dissoutes polluantes peuvent être rejetées dans l'environnement et être transférées dans le sous-sol par infiltration directe ou par le biais de fuites dans des ouvrages de rétention d'eau. Les macropores ou fissures dans les sols peuvent être le lieu de transport accéléré de polluants, par le biais d'un effet de contournement des flux d'eau dans ceux-ci. De plus, le transport accéléré de polluants limite leur possible absorption dans les sols et accroît ainsi le potentiel polluant d'une source dans les 
sols hétérogènes. Les macropores sont créés dans les sols après le passage de petits animaux fouisseurs (vers de terre), par la progression puis le retrait des racines de plantes, ou par fracturation par effets de déformation des sols (déshydratation par exemple) [Beven et Germann, 1982]. De nombreux macropores (notamment ceux provoqués par les vers de terre) sont positionnés verticalement dans les sols et peuvent faire jusqu'à plusieurs mètres. Ils peuvent accélérer le transport de substances polluantes dans les sous-sols et ainsi polluer des nappes d'eau potable [Akay et al., 2008]. On connaît relativement bien les processus de transport dans les milieux homogènes. En effet, l'écoulement est généralement uniforme dans de tels milieux et peut être modélisé à l'aide de la loi de Richards en milieu variablement saturé ou de la loi de Darcy en milieu saturé. Cependant pour les milieux poreux hétérogènes, il est nécessaire de prendre en compte des paramètres liés aux hétérogénéités et les modèles de transport sont multiples. Les modèles double ou multicontinus sont couramment utilisés dans la littérature pour le transport dans les milieux hétérogènes [Köhne et al., 2009]. Les modèles MIM (Mobile/Immobile Model) séparent le milieu poreux en deux sous-domaines d'écoulement, avec un sous-domaine dans lequel l'eau est supposée immobile. Les modèles DPM (Double Permability Model), séparent l'eau en deux sous-domaines avec leur propre perméabilité : la perméabilité de la matrice poreuse et celle de la fracture. Il apparaît d'études antérieures [Ghodrati et al., 1999 ; Lamy et al., 2009] que le chemin préférentiel à forte perméabilité ne peut pas être restreint aux frontières du macropore. De plus les modèles double-perméabilités utilisent des écoulements darciens pour les deux domaines de conductivités différentes, or cette approximation est discutable pour un écoulement dans un macropore. Dans cette étude nous proposons de comprendre les mécanismes hydrodynamiques et hydrodispersifs de l'action du macropore dans le processus de transport de soluté en condition saturée pour un écoulement newtonien en utilisant des colonnes de sol modèles constituées de billes de verre. Nous cherchons pour cela à étudier l'effet de l'influence du débit sur la percée d'un traceur de l'eau. Les courbes de percée obtenues sont utilisées avec un modèle MIM pour déterminer les paramètres hydrodispersifs du transport tels que les volumes impliqués dans le transport, les coefficients de diffusion et le taux d'échange de masse entre sous-domaines. De plus, un modèle numérique basé sur la méthode de Boltzmann sur réseau permet de comprendre les échanges d'eau entre le macropore et le milieu poreux grâce aux simulations numériques à l'échelle des pores.

\section{REVUE BIBLIOGRAPHIQUE}

Afin de modéliser l'écoulement et le transport dans les milieux poreux hétérogènes, on peut décrire les modèles de double porosité et de double perméabilité conceptualisant l'action du macropore comme un chemin préférentiel. Les modèles classiques de double porosité ou perméabilité suggèrent de subdiviser le milieu poreux en deux sous-domaines : un domaine associé à la matrice poreuse, l'autre au macropore. Un modèle double porosités (ou mobile/immobile model MIM) couramment utilisé dans la littérature consiste à considérer deux domaines d'eau liés à ceux précédemment cités : l'un dans lequel l'eau est mobile (englobant le macropore) obéit à l'équation de Richards et l'autre immobile (pour la microporosité, en supposant que celle-ci ne participe pas à l'écoulement) est associé à une équation d'advection-diffusion (CDE) pour le transport. Un coefficient d'échange diffusif du premier ordre entre les deux domaines constitue le taux d'échange lié au gradient de concentration entre ces deux sous-domaines. Ce modèle a été conçu par van Genuchten et Wierenga, [1976] et Gaudet el al. [1977]. On a ainsi l'équation de variation de la concentration en soluté dans la partie mobile et immobile :

$$
\left\{\begin{array}{l}
\theta_{\mathrm{m}} \frac{\partial \mathrm{C}_{\mathrm{m}}}{\partial \mathrm{t}}+\theta_{\mathrm{im}} \frac{\partial \mathrm{C}_{\mathrm{im}}}{\partial \mathrm{t}}=-\theta_{\mathrm{m}} \nabla\left(\mathrm{C}_{\mathrm{m}} \overrightarrow{\mathrm{q}}-\mathrm{D} \nabla \mathrm{C}_{\mathrm{m}}\right) \\
\theta_{\mathrm{m}} \frac{\partial \mathrm{C}_{\mathrm{im}}}{\partial \mathrm{t}}= \pm\left(\mathrm{C}_{\mathrm{m}}-\mathrm{C}_{\mathrm{im}}\right)
\end{array}\right.
$$

avec $\theta_{\mathrm{m}}$ la teneur en eau mobile, $\theta_{\mathrm{im}}$ la teneur en eau immobile, $\mathrm{C}_{\mathrm{m}}$ la concentration dans la phase mobile, $\mathrm{C}_{\mathrm{im}}$ la concentration dans la phase immobile, $\vec{q}$ le vecteur vitesse et $\mathrm{D}$ le coefficient de dispersion. La première équation (1) montre l'évolution de la concentration en soluté dans les domaines d'eaux mobiles et immobiles qui dépendent de la convection avec le vecteur vitesse $\vec{q}$ et de la dispersion hydrodynamique qui, dans un milieu poreux, est l'association de la diffusion moléculaire et de la dispersion mécanique induite par la complexité du cheminement de l'eau dans les pores. L'équation (2) montre l'évolution de la concentration dans le domaine immobile, avec l'influence unique du gradient de concentration entre les deux domaines proportionnellement à un coefficient d'échange de masse $\alpha$. D'autres modèles de double perméabilité (DPM) initialement proposés pour l'industrie des roches réservoirs Barenblatt et al. [1960] séparent le milieu en deux milieux poreux avec pour chacun un écoulement darcien avec sa propre conductivité hydraulique. Le modèle DPM de Gerke et van Genuchten [1993a] d'écoulement et de transport pour les milieux poreux variablement saturés donne l'écoulement comme un couplage de l'équation de Richards pour les deux milieux.

$$
\left\{\begin{array}{l}
\mathrm{c}_{\mathrm{f}} \frac{\partial \mathrm{h}_{\mathrm{f}}}{\partial \mathrm{t}}=\nabla \cdot\left(\overline{\overline{\mathrm{K}_{\mathrm{f}}}} \nabla \mathrm{h}_{\mathrm{f}}\right)+\nabla \cdot\left(\overline{\overline{\mathrm{K}_{\mathrm{f}}}} \nabla \mathrm{z}\right)-\frac{\Gamma_{\mathrm{w}}}{\mathrm{w}_{\mathrm{f}}} \\
\mathrm{c}_{\mathrm{m}} \frac{\partial \mathrm{h}_{\mathrm{m}}}{\partial \mathrm{t}}=\nabla \cdot\left(\overline{\overline{\mathrm{K}_{\mathrm{m}}}} \nabla \mathrm{h}_{\mathrm{m}}\right)+\nabla \cdot\left(\overline{\overline{\mathrm{K}_{\mathrm{m}}}} \nabla \mathrm{z}\right)+\frac{\Gamma_{\mathrm{w}}}{\mathrm{w}_{\mathrm{m}}}
\end{array}\right.
$$

h est la charge hydraulique, $\overline{\bar{K}}$ le tenseur de conductivité hydraulique, c la capacité hydraulique spécifique (les indices $\mathrm{m}$ pour le domaine microporé et $\mathrm{f}$ le domaine fissuré ou macroporé), $\mathrm{z}$ la coordonnée verticale prise vers le haut, $\mathrm{w}_{\mathrm{f}}$ la proportion volumétrique relative du macropore, et $\Gamma_{w}$ le terme de transfert pour les transferts d'eau entre les deux systèmes poreux. On considère que ce terme d'échange est proportionnel à la différence des charges hydrauliques entre les deux domaines :

$$
\Gamma_{\mathrm{w}}=\alpha_{\mathrm{w}}\left(\mathrm{h}_{\mathrm{f}}-\mathrm{h}_{\mathrm{m}}\right)
$$

$\alpha_{w}$ est le coefficient de transfert de premier ordre. En condition saturée, l'écoulement étant considéré permanent, il y a égalité des gradients hydrauliques. Le transfert hydraulique entre les deux domaines est donc considéré nul. Le transport de soluté dans un milieu à double perméabilité est gouverné par deux équations couplées d'advection-dispersion :

$$
\left\{\begin{array}{l}
\frac{\partial}{\partial t}\left(\theta_{\mathrm{f}} R_{\mathrm{f}} \mathrm{C}_{\mathrm{f}}\right)=\nabla \cdot\left(\theta_{\mathrm{f}} \overline{\overline{\mathrm{D}_{\mathrm{f}}}} \nabla \mathrm{C}_{\mathrm{f}}\right)-\nabla \cdot\left(\overrightarrow{\mathrm{q}} \mathrm{C}_{\mathrm{f}}\right)-\theta_{\mathrm{f}} \mu_{\mathrm{f}} \mathrm{C}_{\mathrm{f}}-\frac{\Gamma_{\mathrm{s}}}{\mathrm{w}_{\mathrm{f}}} \\
\frac{\partial}{\partial \mathrm{t}}\left(\theta_{\mathrm{m}} \mathrm{R}_{\mathrm{m}} \mathrm{C}_{\mathrm{m}}\right)=\nabla \cdot\left(\theta_{\mathrm{m}} \overline{\overline{\mathrm{D}_{\mathrm{m}}}} \nabla \mathrm{C}_{\mathrm{m}}\right)-\nabla \cdot\left(\overrightarrow{\mathrm{q}_{\mathrm{m}}} \mathrm{C}_{\mathrm{m}}\right)-\theta_{\mathrm{m}} \mu_{\mathrm{m}} \mathrm{C}_{\mathrm{m}}+\frac{\Gamma_{\mathrm{s}}}{\mathrm{w}_{\mathrm{m}}}
\end{array}\right.
$$

Avec $\theta_{\mathrm{f}}$ et $\theta_{\mathrm{m}}$ les teneurs en eau dans le macropore et dans la matrice poreuse respectivement, $\mathrm{C}$ la concentration en soluté, $\mathrm{R}$ le facteur de retard, $\mu$ un coefficient de décroissance et $\Gamma_{\mathrm{s}}$ le terme de transfert de masse évalué par Gerke et van Genuchten [1993b] :

$$
\Gamma_{\mathrm{s}}=+\Gamma_{\mathrm{w}} \mathrm{C}_{\mathrm{f}}+\alpha_{\mathrm{s}} \mathrm{w}_{\mathrm{m}} \theta_{\mathrm{m}}\left(\mathrm{C}_{\mathrm{f}}-\mathrm{C}_{\mathrm{m}}\right)
$$


Avec $\alpha_{\mathrm{s}}$ le coefficient de transfert de masse de premier ordre. Le transfert de masse peut ainsi être défini comme la somme de la contribution de l'aspect diffusif (deuxième terme de droite) et d'un terme advectif lié au transfert de fluide entre les deux domaines $\Gamma_{w}$.

Cependant des mesures de courbes de percée d'injection de traceur sur colonne figurant l'évolution temporelle de la concentration d'un traceur en sortie de colonne montrent que ces modèles sous-estiment l'action du macropore en contraignant le flux préférentiel au domaine du macropore. Ainsi, l'augmentation de la conductivité hydraulique ne peut se limiter aux frontières de celui-ci. Ghodrati et al. [1999] ont montré expérimentalement à l'aide de l'injection de colorant que l'écoulement dans la microporosité est renforcé autour du macropore par la présence de celui-ci. De la même manière, Lamy et al. [2009] ont montré qu'une action du macropore (limitée numériquement à son emplacement physique) sur les phénomènes de transport est en désaccord avec les courbes de percées expérimentales. Des courbes de percées expérimentales de traceur non réactif $(\mathrm{KBr})$ dans une colonne de sable avec un macropore montrent une inflexion avec un premier pic important correspondant au passage du traceur principalement dans le macropore et un deuxième pic plus faible, correspondant au restant du traceur passant par la matrice poreuse. Cependant, en utilisant un modèle DPM couplé à une approche MIM avec ajustement du rapport des conductivités hydrauliques entre la matrice poreuse et le macropore, des coefficients de dispersion longitudinale et transversale, et de la teneur en eau immobile, en condition saturée, les courbes de percées modélisées montrent un premier pic précoce plus faible que le second ce qui suggère que l'impact du macropore sur le transport préférentiel est sous-estimé. En élargissant le domaine de plus forte perméabilité du macropore (la zone préférentielle) à une section allant de $1 \%$ à $50,5 \%$ de la section totale de la colonne et en contraignant un rapport de 4 pour le rapport de la conductivité hydraulique du macropore sur celle de la matrice poreuse, un meilleur ajustement a été trouvé avec l'apparition d'un premier grand pic correspondant au transport dans le chemin préférentiel et un deuxième plus faible correspondant au passage dans la matrice poreuse non influencée par le macropore. Cette étude révèle que la dispersion longitudinale est plus importante dans la zone préférentielle que dans la matrice poreuse et que la dispersivité transversale entre la zone préférentielle et la matrice poreuse explique la baisse lente de concentration. La modification du champ d'écoulement est prise en compte dans certains modèles par l'utilisation de la loi de Poiseuille pour le macropore car il n'y a pas de forces capillaires dans celui-ci [Malone et al., 2004].

Pour mieux comprendre les mécanismes d'écoulement et de transport dans les milieux à géométrie complexe, il est nécessaire de comprendre les champs d'écoulement à l'échelle des pores et notamment dans la zone de la matrice poreuse à la périphérie du macropore. La méthode de Boltzmann sur réseau (LBE) est une méthode numérique couramment utilisée en hydrodynamique car elle utilise un algorithme simple et peut être utilisée pour simuler des écoulements newtoniens et non newtoniens. Elle permet d'obtenir une approximation discrète de la solution de l'équation de Navier-Stokes et permet d'obtenir les champs de vitesse et de pression [Succi, 2001]. Une méthode LBE-BKG [Bhatnagar et al., 1954] dispose d'un paramètre de relaxation $\tau$ pour le terme de collision. On obtient l'évolution de la densité de masse $\mathrm{f}_{\mathrm{i}}(\mathrm{r}, \mathrm{t})$ à un nœud $\mathbf{r}$ dans la direction $\mathrm{c}_{\mathrm{i}}$ du nœud voisin $\mathrm{r}+\mathrm{c}_{\mathrm{i}}$ avec

$$
f_{i}\left(\vec{r}+\vec{r}_{i}, t+1\right)=(1-\tau) f_{i}(\vec{r}, t)+\tau f_{i}^{e q}(\vec{r}, t)
$$

avec $\mathrm{f}_{\mathrm{i}}^{\mathrm{eq}}$ la fonction de distribution à l'équilibre donnée pour le modèle BGK par Qian et al. [1992].

\section{MÉTHODES EXPÉRIMENTALES ET APPROCHES NUMÉRIQUES}

\section{III.1. Colonnes expérimentales}

Les colonnes expérimentales $(14,5 \mathrm{~cm}$ de longueur pour un diamètre de $5 \mathrm{~cm}$ ) sont composées de billes de verre (distribution continue de tailles de grains de $425 \mu \mathrm{m}$ à $800 \mu \mathrm{m}$ ) afin de limiter les effets d'adsorption. Les billes sont collées entre elles avec de l'araldite et le macropore est créé en insérant une tige en Téflon rectiligne de $0,3 \mathrm{~cm}$ de diamètre avant de déposer les grains enduit de colle dans la colonne puis extrait de celle-ci lorsque le milieu est en phase de consolidation. Une colonne sans macropore fait office de colonne témoin. Des mesures de courbes de percée ont été effectuées avec une solution traçante de bromure de potassium $(\mathrm{KBr})$. Le traçage consiste en l'injection en continu d'une solution de conditionnement de $\mathrm{KBr}$ de concentration $5.10^{-4}$ mol. $\mathrm{L}^{-1}$ et l'injection d'un créneau de $5 \mathrm{~mL}$ de solution traçante de $\mathrm{KBr}$ de concentration $5.10^{-3}$ mol.L $\mathrm{L}^{-1}$. L'injection se fait à débit constant sans interruption lors de l'injection du traceur. Les courbes de percée ont été obtenues soit par mesure en continu de la conductivité électrique en sortie de colonne (Aktaprime, GE Healthcare Bio-Sciences), soit par mesure directe des concentrations de sortie en ions bromure par chromatographie ionique (THERMO Scientific) ou en gadolinium par spectrométrie de masse (ICP MS VARIAN 820-MS) et/ou RMN (Bruker). Ces courbes permettent de déterminer les bilans de masse et les facteurs de retard en utilisant la méthode des moments [Lamy, 2008]. De plus, les paramètres hydrodispersifs caractérisant l'écoulement et le transport d'un modèle MIM (équations (1) et (2)) sont déterminés par inversion des données expérimentales grâce au logiciel HYDRUS-1D [Simunek et al., 1998]. Ces mesures expérimentales ont été effectuées en condition saturée, à différents débits d'injection (de $0,05 \mathrm{~mL} \cdot \mathrm{min}^{-1}$ à $5,0 \mathrm{~mL} \cdot \mathrm{min}^{-1}$ ) afin d'étudier l'influence du débit sur la percée dans la colonne avec macropore. Les mesures de conductivité électrique ont été normalisées par la conductivité de la solution traçante. De plus, les volumes injectés sont normalisés avec $\mathrm{V}_{0}$ correspondant au volume poreux des colonnes. Par ailleurs, les volumes morts sont retirés. Ceux-ci correspondent aux volumes des tuyaux entre la valve d'injection et l'entrée de la colonne, ainsi que les volumes partant de la sortie de la colonne jusqu'à la cellule de mesure de conductivité électrique. Des mesures plus qualitatives de visualisation en deux dimensions de transfert de traceur paramagnétique (Gd-DTPA) dans les colonnes ont été effectuées par IRM en utilisant des séquences double écho de spin pondérées par le temps de relaxation $T_{1}$. Ce traceur est couramment utilisé en imagerie médicale car il ne présente pas de toxicité particulière pour l'organisme. Il est de plus très adapté pour l'imagerie de transport dans les milieux poreux [Habert-Pohlmeier et al., 2010]. Ces mesures permettent de suivre l'évolution en deux dimensions du traceur au sein des colonnes et ainsi d'établir une cartographie de la concentration en traceur.

\section{III.2. Modélisations numériques}

Des simulations d'écoulement et de transport de soluté ont été effectuées avec la méthode de Boltzmann sur réseaux à un temps de relaxation (LBE-BGK) (équation (9)) avec le 
$\mathrm{F}_{\mathrm{i}}^{\mathrm{eq}}$ de Qian et al., [1992] qui permet de résoudre numériquement l'équation de Navier-Stokes, afin de mieux comprendre l'écoulement à l'interface entre le milieu poreux et le macropore à l'échelle des pores. Un réseau D2Q9 (milieu en deux dimensions avec 9 vitesses discrètes par nœud) est utilisé pour cette étude [Qian et D'Humières, 1992]. Le réseau de grains est constitué de cercles placés aléatoirement et ayant une distribution continue de tailles comprise entre deux valeurs ayant un rapport de 1,06 entre elles. Trois réseaux de grains sont définis avec les tailles moyennes de grains différentes (Tableau 1).

Tableau 1 : Réseaux poreux utilisés pour les simulations d'écoulement avec la méthode de Boltzmann sur réseau, avec l'étendue de la taille des grains et la porosité.

\begin{tabular}{|c|c|c|}
\hline Nom réseau & Tailles grains $(\mathbf{c m})$ & Ønum \\
\hline 1 & $0,60-0,63$ & $47,4 \%$ \\
\hline 2 & $0,42-0,45$ & $47,0 \%$ \\
\hline 3 & $0,33-0,36$ & $43,2 \%$ \\
\hline
\end{tabular}

Pour la création du milieu poreux numérique, à chaque grain, placé initialement sur un réseau cristallin, est défini un vecteur vitesse selon une loi gaussienne puis s'enchaînent un nombre de collisions élastiques supérieur à 1000 afin de disposer les grains de façon aléatoire. Le matériau numérique obtenu est compacté avec un chargement isotrope. Puis chaque rayon des grains est réduit d'un facteur 0,8 afin de créer un domaine poreux connecté en deux dimensions. Les influences de la taille du macropore et de la taille moyenne des grains sont étudiées.

\section{RÉSULTATS ET DISCUSSION}

Les courbes de percée normalisées de bromure de potassium de la colonne témoin (Figure 1 (a)) ne montrent aucune influence du débit d'injection sur la forme de la percée. La percée pour tout débit est quasiment symétrique entre la montée et la descente. La percée du traceur débute à peu près à $0,5 \mathrm{~V}_{0}$ et atteint son maximum à $1 \mathrm{~V}_{0}$. Le maximum de concentration en sortie correspond au dixième de la concentration initiale du traceur. Il faut environ 2,5 volumes poreux pour évacuer tout le traceur pour tous les débits. Les bilans de masse (BM) et facteur de retard (R) calculés par la méthode des moments pour toutes les courbes de percée sont très proches de 1 , indiquant que tout le traceur est évacué de la colonne et qu'il a visité un volume équivalent à la totalité du volume poreux de la colonne. En revanche, les percées de la colonne macroporée (Figure 1 (b)) montrent une forte variation du comportement de l'élution du traceur en fonction du débit injecté. En effet, pour un débit suffisamment faible (inférieur à $0,2 \mathrm{~mL} \cdot \mathrm{min}^{-1}$ ) apparaît une inflexion de plus en plus visible à mesure que le débit diminue, dans la percée des ions bromures. Tandis que pour un fort débit (supérieur à $0,5 \mathrm{~mL} \cdot \mathrm{min}^{-1}$ ) il n'apparaît qu'un pic avec une forte asymétrie entre la montée et la descente. L'élution du traceur apparaît dès le début de l'injection et devient négligeable avant $0,5 \mathrm{~V}_{0}$ pour tous les débits. Les valeurs maximums de concentrations atteignent $80 \%$ de la concentration du traceur pour un fort débit et diminuent à mesure que celui-ci baisse.

Les inversions des données expérimentales peuvent nous donner un indice sur les volumes impliqués dans le transport, les volumes immobiles, les coefficients de dispersion et le coefficient d'échange de masse entre les deux domaines pour le modèle MIM. Les résultats sont dans le Tableau 2. À mesure que le débit diminue le volume visité par le traceur augmente (d'un facteur 10 pour une diminution de débit d'un facteur 50). Pour rappel, volume du macropore est de $1,02 \mathrm{~mL}$. À fort débit, le traceur traverse un volume du même ordre de grandeur que le macropre. De plus, les volumes immobiles et mobiles dans l'hypothèse MIM augmente fortement lorsque le débit diminue. La dispersion augmente également d'un facteur 10 entre les valeurs extrêmes du débit et le coefficient d'échange de masse entre les eaux mobile et immobile diminue d'un facteur 40 .

Ainsi, à mesure que le débit diminue, la dispersion augmente et permet la visite d'un volume plus important du milieu poreux. Comme le suggère Lamy et al. [2009] l'inflexion des courbes de percée peut être expliquée par une extension du chemin préférentiel sur un volume dépassant celui du macropore. À fort débit le volume impliqué par le transport est du même ordre de grandeur que le volume du macropore. Ainsi la zone préférentielle se limite au macropore et implique peu le milieu poreux environnant

En effectuant les mêmes types de traçage sur un tube de mêmes dimensions que le macropore, afin de contraindre l'écoulement à celui-ci, on obtient la Figure 2 (a) qui montre qu'à faibles débits (inférieurs à $0,2 \mathrm{~mL} \cdot \mathrm{min}^{-1}$ ) les effets diffusifs sont dominants et ne déforment la forme du créneau
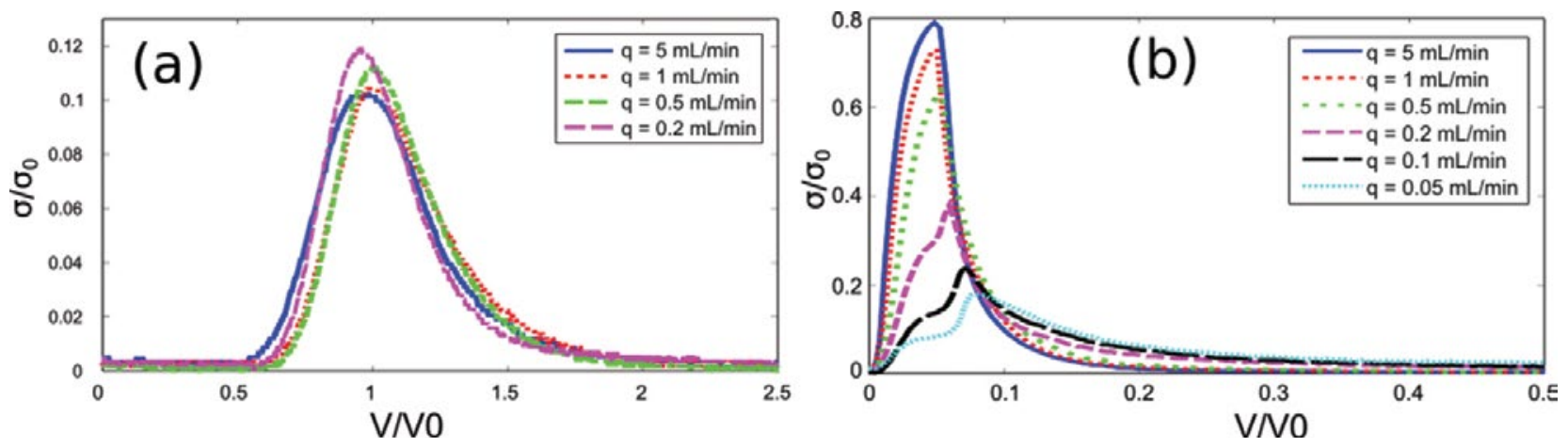

Figure 1 : Courbes de percée du KBr dans la colonne témoin (a), et la colonne macroporée (b), suivant différents débits d'injections. 
injecté qu'au début et à la fin de l'injection. En revanche à fort débit (supérieur à $0,5 \mathrm{~mL} \cdot \mathrm{min}^{-1}$ ) la forme du créneau d'injection disparaît et la percée perd sa symétrie à cause d'effets convectifs plus importants dans le tube. En comparant les percées du $\mathrm{KBr}$ dans le tube simulant le macropore et la colonne macroporée (Figure 2 (b)), on constate qu'à fort débit $\left(5,0 \mathrm{~mL} \cdot \mathrm{min}^{-1}\right)$ les percées sont similaires dans la montée et la descente, tandis qu'à faible débit $\left(0,1 \mathrm{~mL} \cdot \mathrm{min}^{-1}\right)$, l'écart important entre les deux percées peut s'expliquer par un échange latéral important avec la matrice poreuse causé par une diffusion plus importante entre le macropore et la matrice poreuse environnante.

Le coefficient de diffusion de l'ion bromure dans l'eau pure à $20^{\circ} \mathrm{C}$ est de $2,08.10^{-9} \mathrm{~m}^{2} \cdot \mathrm{s}^{-1}$ [Robinson et Stokes, 1960]. La forte diffusivité latérale du $\mathrm{KBr}$ dans l'eau peut expliquer l'apparition de l'inflexion de la percée pour la colonne macroporée. L'ajout d'un agent visqueux comme le glycérol fait baisser le coefficient de diffusion de l'ion bromure dans la solution, en considérant la relation de Stokes-Eisntein qui indique que le coefficient de diffusion moléculaire du soluté est inversement proportionnel à la viscosité dynamique du liquide. On abaisse le coefficient de diffusion de l'ion bromure pour qu'il atteigne une valeur du même ordre de grandeur que celui du Gd-DTPA dans l'eau. Pour cela, il faut rajouter $50 \%$ de glycérol (en masse) dans la solution. La viscosité de la solution passe de de $1,0.10^{-3}$ Pa.s à environ $6,0.10^{-3} \mathrm{~Pa}$.s et la densité de 1 à 1,13 . En effectuant des courbes de percée dans cette solution il faut prendre en compte la différence de viscosité qui influe sur l'écoulement pour un débit identique dans l'eau. En effet, le nombre de Reynolds est proportionnel à la vitesse avec un rapport $\rho / \mu$. Ce rapport devient 5,13 fois plus faible lors de l'ajout du glycérol dans la solution. Ainsi pour respecter la similarité des écoulements, on doit multiplier les débits étudiés dans l'eau pure par 5 environ dans le mélange eau/ glycérol pour obtenir le même écoulement.

Le débit de $0,1 \mathrm{~mL} \cdot \mathrm{min}^{-1}$ dans l'eau a le même nombre de Reynolds que le débit de $0,5 \mathrm{~mL} \cdot \mathrm{min}^{-1}$ dans la solution eau/glycérol. La mesure de conductivité est altérée par la présence de glycérol, celle-ci est 10 fois plus faible que celle obtenue dans une solution à $100 \%$ d'eau dans les mêmes conditions d'injection, ainsi la mesure de concentration en ion bromure est effectuée par spectrométrie de masse. On observe ainsi sur les courbes de percée du bromure en Figure 3 que l'inflexion n'apparaît pas (dans les mêmes conditions, pour le bromure dans une solution d'eau, l'inflexion est visible).
Les images IRM de l'injection du traceur paramagnétique Gd-DTPA dans la colonne macroporée obtenue avec une séquence double écho de spin pondérée en $\mathrm{T}_{1}$ à un débit de $0,10 \mathrm{~mL} \cdot \mathrm{min}^{-1}$ (Figure 4) montrent bien le passage du soluté dans le macropore, ainsi qu'une légère dispersion latérale dans la porosité environnante. On aperçoit de plus que le traceur reste piégé un certain temps dans la porosité, cela est dû au phénomène de contournement du flux. En effet, une grande part du débit passe dans le macropore. Ainsi le débit dans la porosité est réduit et inférieur à celui dans la colonne homogène pour un même débit d'injection. Des courbes de percée ont été obtenues à différents débits avec du Gd-DTPA (non présentées ici) et n'ont pas montré l'apparition d'une inflexion pour des faibles débits comme pour le KBr. Le coefficient de diffusion du Gd-DTPA dans une solution libre est de $4,0.10^{-10} \mathrm{~m}^{2} \cdot \mathrm{s}^{-1}$ [Foy et Blake, 2001], soit cinq fois inférieur à celui du bromure dans l'eau. Les résultats de percée du Gd-DTPA et du $\mathrm{KBr}$ avec le glycerol sont similaires avec une courbe monomodale pour tous les débits testés. Ainsi sur ces images IRM, l'inflexion, visible avec le bromure, ne peut pas être expliquée. Cependant, la Figure 4 met en évidence un phénomène de transport latéral de plus en plus important à mesure que le débit baisse. On peut en déduire avec les Figures 3 et 4 que le coefficient de diffusion est, avec le débit, déterminant pour l'apparition d'une inflexion. Le transfert diffusif latéral important d'un traceur au coefficient de diffusion relativement élevé peut être la cause de l'apparition d'une inflexion.

\section{IV.1. Modélisations directes des écoulements}

Les simulations d'écoulement par la méthode de Boltzmann sur réseau BGK en deux dimensions sur un milieu poreux granulaire ont permis de mettre en évidence le champ de vitesse moyen d'un milieu poreux macroporé en fonction de la distance à l'axe du macropore. Différentes tailles de macropore ont été testées pour un même débit d'injection. De plus, ces différentes simulations ont été effectuées sur les trois différents milieux poreux listés en Tableau 1. On peut voir en Figure 5 (a) que l'influence du macropore sur la vitesse moyenne d'écoulement dépasse ses frontières et qu'il existe une zone à écoulement préférentiel dans la matrice poreuse comme le suggèrent Beavers et Joseph [1967] avec une condition de glissement aux frontières du macropore. Cette zone a une dimension du même ordre de grandeur que la taille moyenne des grains mais ne dépend pas de la taille du macropore (Figure 5 (b)).
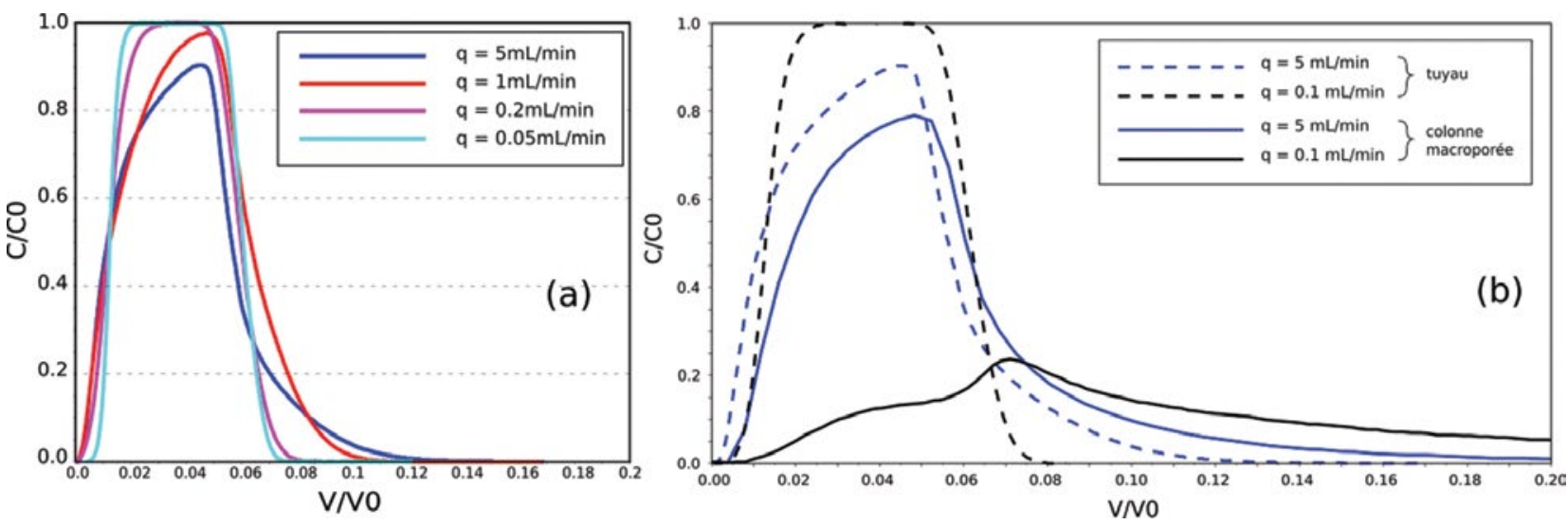

Figure 2 : (a) Courbes de percée du KBr dans un tube de mêmes dimensions que le macropore. (b) Comparaison des courbes de percée de la colonne macroporée (en traits pleins) et du tube (en traits pointillés) aux mêmes débits. 


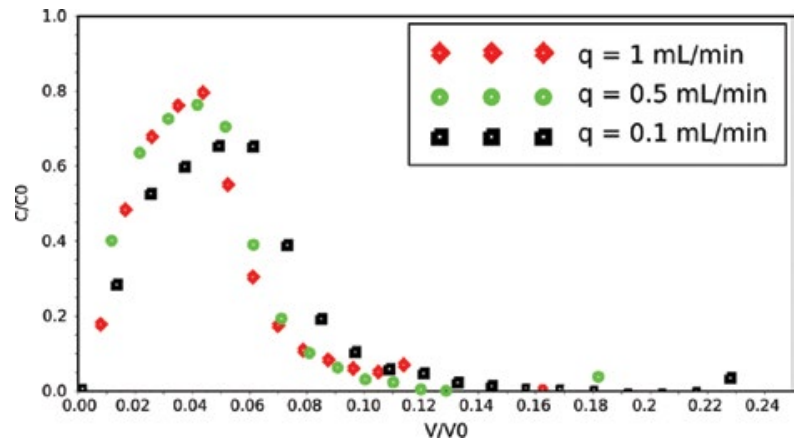

Figure 3 : Courbes de percée du KBr dans la colonne macroporée dans une solution composée d'un mélange d'eau ultrapure et de glycérol (50\% en masse).

L'écoulement dans le macropore crée une contrainte de cisaillement qui est transmise dans la matrice poreuse. La forme de l'écoulement dans le macropore est de type Poiseuille pour toute taille de macropore comme dans le modèle de Beavers et Joseph [1967].

\section{CONCLUSIONS ET PERSPECTIVES}

Cette étude se propose d'étudier le comportement à l'échelle des pores de l'écoulement dans un milieu poreux traversé par un macropore et de caractériser l'apparition d'une inflexion sur les percées en-dessous d'un certain débit d'injection. L'influence du macropore se caractérise par le transport préférentiel du soluté à travers celui-ci. À faible débit, on peut noter un échange diffusif du macropore vers la matrice poreuse environnante qui peut justifier l'inflexion des courbes de percée. De plus l'imagerie, montre un échange du macropore vers la matrice poreuse mais ne montre pas de progression par advection du traceur présent dans la porosité, suggérant le rôle important de la diffusion pour le transport. Les simulations d'écoulement montrent que le chemin préférentiel est étendu sur une zone de la taille moyenne des grains. On a ainsi un comportement d'échange perte/gain de masse du macropore vers lui-même justifiant la prise en compte d'une zone élargie d'écoulement et de transport de soluté dans la zone de transport macroporale. Cette zone est néanmoins de taille plus faible que proposée par Lamy et al [2009]. Ces résultats montrent
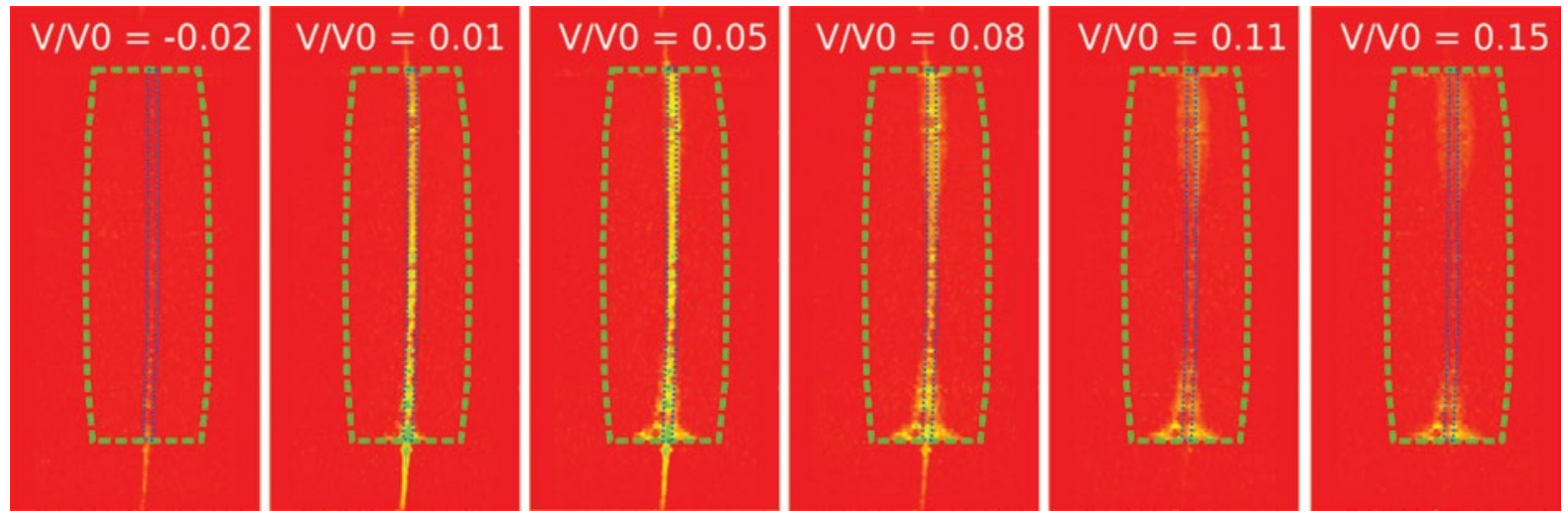

Figure 4 : Imagerie par RMN (signal $T_{1}$ ) de l'injection de $5 \mathrm{~mL}$ de solution paramagnétique (Gd-DTPA) dans la colonne macroporée. L'indice $V / V_{0}$ indice le volume normalisé injecté avec la valeur 0 correspondant au début de l'entrée du traceur dans la colonne. Les flèches blanches montrent la progression de l'étendue du traceur dans la matrice poreuse.
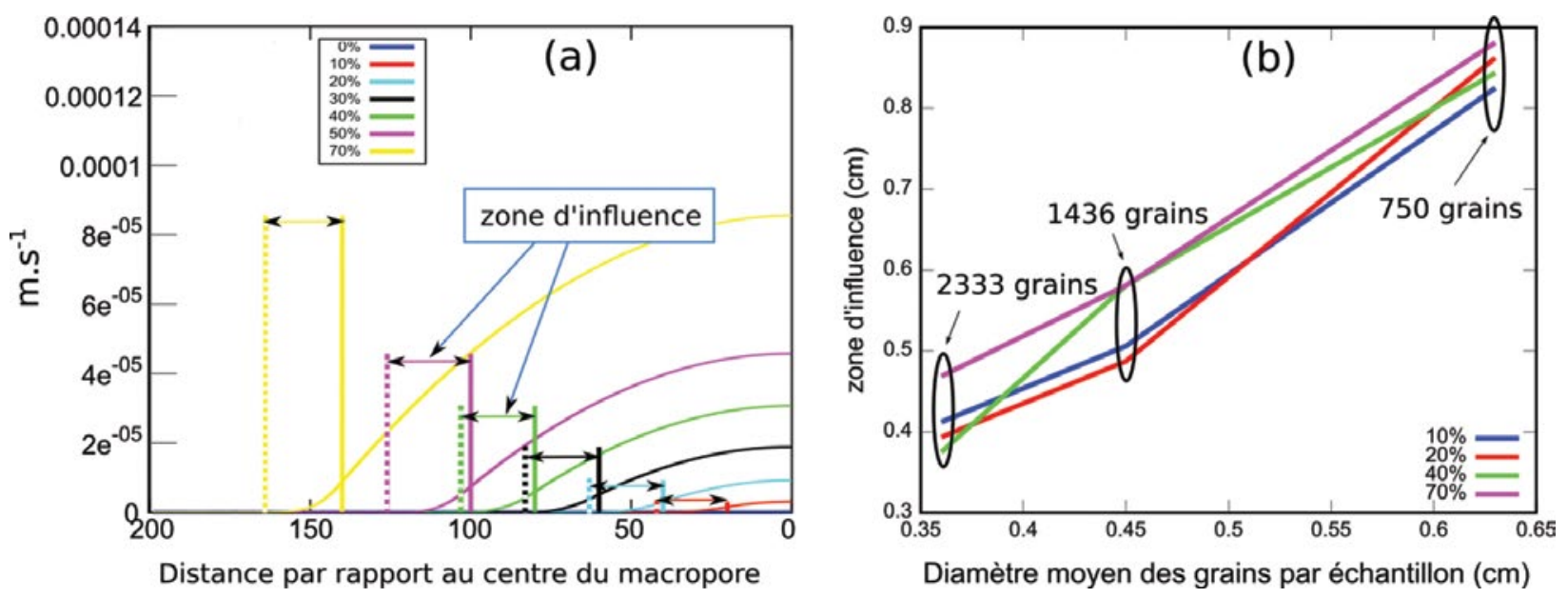

Figure 5 : (a) Champ de vitesse moyen simulé par LBE-BGK pour le milieu poreux $n^{\circ} 1$ (Tableau 1) avec différentes tailles de macropore. (b) Taille des zones d'écoulement préférentiel dans la matrice poreuse en fonction de la taille du macropore et de la distribution des grains. 
que le macropore influe sur la porosité environnante sur une zone de la taille des grains pour l'écoulement et sur une zone dépendante du coefficient de diffusion du traceur ainsi que du temps de séjour moyen de celui-ci pour le transfert des solutés. De nouvelles simulations d'écoulement et de transport sont a effectuer sur les milieux poreux modèles, en utilisant notamment la méthode TRT [Ginzburg, 2008] qui permet d'éviter la dépendance de la viscosité vis-à-vis de la perméabilité. Différents débits seront testés et des courbes de percée modélisées pourront être obtenues.

\section{REMERCIEMENTS}

Les auteurs tiennent à remercier D. Demare, N. Caubrière et C. Ragoin du pôle analytique du laboratoire EE de l'IFSTTAR, ainsi que S. Rodts, D. Courtier-Murias et J. Gil Roca de l'équipe de recherche milieu poreux du laboratoire Navier de l'IFSTTAR.

\section{RÉFÉRENCES}

AKAY O., Fox G. A., \& ŠIMŮNEK J. (2008) - Numerical Simulation of Flow Dynamics during Macropore-Subsurface Drain Interactions Using HYDRUS. Vadose Zone Journal, 7(3), 909.

Barenblatt G., Zheltov I., \& Kochina I. (1960) - Basic concepts in the theory of seepage of homogeneous liquids in fissured rocks. Journal of Applied Mathematics and Mechanics, 24(5).

Beavers G. S., \& Joseph D. D. (1967) - Boundary conditions at a naturally permeable wall. Journal of Fluid Mechanics, 30(1), 197-208.

Beven K., Germann P. (1982) - Macropores and water flow in soils. Water resources research, 18(5), 1311-1325.

Bhatnagar P. L., Gross E. P., \& Krook M. (1954) - A model for collision processes in gases. I. Small amplitude processes in charged and neutral one-component systems. Physical Review, 94(3), 511-525.

FoY B. D., \& BlaKe J. (2001) - Diffusion of paramagnetically labeled proteins in cartilage: enhancement of the 1-D NMR imaging technique. Journal of Magnetic Resonance, 148(1), 126-134.

Gaudet J. P., Jégat H., Vachaud G., \& Wierenga P. J. (1977) Solute Transfer, with Exchange between Mobile and Stagnant Water, through Unsaturated Sand. Soil Science Society of America Journal, 41(4), 665-671.

Gerke H. H., Van Genuchten M. Th. (1993) - A dual-porosity model for simulating the preferential movement of water and solute in structured porous media. Water Resources research, 29(2), 305-319.
Gerke H. H., \& VAn Genuchten M. T. (1993) - Evaluation of a first-order water transfer term for variably saturated dual-porostity flow models. Water Resources Research, 29(4), 1225-1238.

Ginzburg I., Verhaeghe F., D'humières D. (2008) Two-Relaxation-Time Lattice Boltzmann Scheme: About Parametrization, Velocity, Pressure and Mixed Boundary Conditions. Communications in Computational Physics, 3(2), 427-478.

Ghodrati M., Chendorain M., \& Chang Y. J. (1999) Characterization of macropore flow mechanisms in soil by means of a split macropore column. Soil Science Society of America Journal, 63(5), 1093-1101.

Habert-Pohlmeier S., Bechtold M., Stapf S., \& Pohlmeier A. (2010) - Water flow monitored by tracer transport in natural porous media using magnetic resonance imaging. Vadose Zone Journal, 2(9).

KöHNE J. M., KöHNE S., \& SimunEK J. (2009) - A review of model applications for structured soils: a) Water flow and tracer transport. Journal of Contaminant Hydrology, 104(1-4), 4-32.

LAMY E. (2008) - Ecoulement et transfert collö̈dal dans des matrices hétérogènes et stratifiées, application à des milieux poreux modèles. (Thèse de doctorat).

Lamy E., Lassabatere L., Bechet B., Andrieu H. (2009) Modeling the influence of an artificial macropore in sandy columns on flow and solute transfer. Journal of Hydrolody, 376, 392-402.

Malone R. W., Ahuja L. R., Ma L., Wauchope R. D., Ma Q., \& Rojas K. W. (2004) - Application of the Root Zone Water Quality Model (RZWQM), to pesticide fate and transport: An overview. Pest Management Science, 60(3), 205-221.

Qian Y. H., D'humieres D., \& Lallemand P. (1992) - Lattice BGK Models for Navier-Stokes Equation. EPL (Europhysics Letters), 17, 479-484.

Robinson R. A., \& Stokes R. H. (1960) - Electrolyte solutions, Dover Publications.

SHAPIRO M., \& BRENNER H. (1990) - Taylor dispersion in the presence of time-periodic convection phenomena. Part II. Transport of transversely oscillating Brownian particles in a plane Poiseuille flow. Physics of Fluids A: Fluid Dynamics, 2(10), 1744-1753.

Simunek J., Sejna M., Van Genuchten M.Th. (1998) - The HYDRUS-1D software package for simulating the one-dimensional movement of water, heat, and multiple solutes in variably saturated media. Version 2.0, IGWMC-TPS-70, International Ground Water Modeling Center, Colorado School of Mines, Golden, Colorado.

Succi S. (2001) - The Lattice Boltzmann Equation: For Fluid Dynamics and Beyond.

Van Genuchten M. Th., Wierenga P. J. (1976) - Mass transfer studies in sorbing porous media 1. Analytical solutions. Soil Science Society of America Journal, 40(4), 473-480. 